Discrete Comput Geom 31:305-321 (2004)

DOI: $10.1007 / \mathrm{s} 00454-003-2865-1$

Geometry

\title{
Intersection and Linking Numbers in Oriented Matroids*
}

\author{
Paula Carvalho $^{1}$ and António Guedes de Oliveira ${ }^{2}$ \\ ${ }^{1}$ R\&D Unit Matemática e Aplicações, Departamento de Matemática, \\ Universidade de Aveiro, Campus de Santiago, \\ P-3810-193 Aveiro, Portugal \\ paulac@mat.ua.pt \\ ${ }^{2}$ Centro de Matemática da Universidade do Porto, \\ Departamento de Matemática Pura, Faculdade de Ciências da U. Porto, \\ Rua do Campo Alegre 687, P-4169-007 Porto, Portugal \\ agoliv@fc.up.pt
}

\begin{abstract}
We introduce for oriented matroids a generalization of the concepts of intersection and linking numbers in Euclidean space, with most of their main properties (see [18]). As an application, we reprove a result of Brehm [8] in a slightly extended form.
\end{abstract}

\section{Introduction}

Since a triangulated orientable combinatorial 2-manifold (viewed here as a simplicial complex) is a closed, orientable (local) surface without boundary, it is well known (see [16]) that it can be embedded in the Euclidean space of dimension 3. Yet, in general, the embedding does not preserve the complex, in the sense that the images of the edges are no longer always straight, and hence the image of the triangles are also no longer always triangles. Whether such a geometric (versus topological) embedding exists is a new problem, with very few answers, even for these dimensions.

In the case when the simplicial complex has genus zero, Steinitz's theorem applies: since the 1-skeleton of the simplicial complex is a "planar, simple, 3-connected graph", it is the graph of a polyhedron, and so the problem has a positive answer in general. For greater genus, the first counterexample to a long standing conjecture by Grünbaum [10], claiming that the same would happen, has genus six and is due to Bokowski and the second author [5]. In fact, it was proved in [5] that, for every integer $g \geq 6$, there is a

\footnotetext{
* This research was partially supported by FCT and PRAXIS XXI through the Research Units Pluriannual Funding Program, and by project 36563/99.
} 
simplicial complex, a triangulation of a closed orientable surface of genus $g$, that cannot be embedded geometrically in 3-space.

The proof in [5], as in various other cases where geometric embeddings were found, was obtained by using oriented matroids. Let $\mathcal{O}$ be the oriented matroid of a given point configuration, let $T$ be a triangle and let $E$ be an edge of the simplicial complex embedded in Euclidean 3-space through the configuration. Then there will be no circuit (a minimal dependent set) of $\mathcal{O}$ with $T$ as the positive part (the points with positive coefficient in the linear dependency) and $E$ as the negative part, for this would mean, by Radon's theorem, $\operatorname{conv}(E) \cap \operatorname{conv}(T) \neq \emptyset$ - and so $E$ would intersect $T$. Hence a proof that there exists no proper oriented matroid admissible [7] with a given abstract simplicial complex, establishes that the complex is not geometrically embeddable in Euclidean 3 -space. This was done in [5], where all possible oriented matroids were generated up to a certain point and tested against the simplicial complex by computer.

Another 2-simplicial complex, which is very interesting in this context, was introduced by Brehm in [8]: it is a non-orientable complex with boundary (a triangulation of a Möbius strip) that also cannot be embedded geometrically in $\mathbb{R}^{3}$. Although the proof in [8] is of a different (topological) nature, the method used in [5] shows in a very short CPUtime that there is also no oriented matroid admissible with its list of triangles [3]. Other topological methods have been tested on these problems. In particular, a very interesting refinement of the methods of Van Kampen and Flores, introduced by Novik [11], fails both for Brehm's and for one of Altshuler's simplicial complexes.

In this introductory paper, our aim is to develop for oriented matroids the standard topological tools of intersection and linking numbers. With these tools we can also manage to prove the non-existence of oriented matroids admissible with a given complex. This is done below (Section 4) for Brehm's triangulation, where we mimicked the original proof by Brehm for this setting, mainly as an example of application-although, in fact, the two papers cited above, [8] and [11], were the starting point for this construction. Since these notions play such an important rôle in the context of topological embeddings of complexes in Euclidean space, we believe they can also be of some use in the setting of oriented matroids, with all its benefits, namely the fact that these notions can be handled by computer.

Furthermore, we believe that the study of the properties of these topological invariants is of much interest in the context of oriented matroids. In particular, we hope that in the future they can help to clarify to what extent oriented matroids generalize Euclidean space, by extending (or not) its properties. In this direction, we prove the following property (see [18]) of the linking number, here denoted by [[-]], which is, in fact, the main result of this paper (see Section 2 for definitions):

Theorem 3.2. Let $\mathcal{O}$ be a (uniform, acyclic) oriented matroid of rank $r$. Let $z_{1}=\partial x_{1}$ and $z_{2}=\partial x_{2}$ be two support-disjoint cycles in $\mathcal{O}$ with $\operatorname{dim} z_{1}=p, \operatorname{dim} z_{2}=q$ and $p+q=r-2$. Then:

(ii) $\left[\left[z_{1}, z_{2}\right]\right]=(-1)^{p q+1}\left[\left[z_{2}, z_{1}\right]\right]$.

This result is an easy consequence of:

Theorem 3.1. Let $\mathcal{O}$ be a (uniform, acyclic) oriented matroid of rank $r$ and let $x$ and $y$ be a p-chain and a q-chain, respectively, in $\mathcal{O}$. Then, using $\mathcal{I}(x, y)$ as the intersection 
number of $x$ and $y$ :

(i) $\mathcal{I}(x, y)=(-1)^{p q} \mathcal{I}(y, x)$ if $p+q=r-1$;

(ii) $\mathcal{I}(\partial x, y)=(-1)^{p} \mathcal{I}(x, \partial y)$ if $p+q=r$.

We follow [1] for definitions and notation in oriented matroids, which we review briefly below.

\section{Main Concepts and Notation}

In this paper we do not distinguish between the two concepts of chirotope and oriented matroid [2]. Indeed, it was proved by Lawrence [15] that any chirotope (a concept introduced by Gutierrez Novoa as a "multiple ordered set" [12]) is the signature of the bases, as defined by Las Vergnas [13], of an oriented matroid and vice versa. We focus on the properties that are used. We start with a motivating example.

Let $\mathcal{P}=\left\{p_{1}, p_{2}, \ldots, p_{n}\right\}$ be a point configuration in $\mathbb{R}^{d}, r=d+1, E=\{1,2, \ldots, n\}$ and $v_{i}=\left(1, p_{i}\right) \in \mathbb{R}^{r}$ for $i \in E$; we call $\chi:\left\{\left(\lambda_{1}, \lambda_{2}, \ldots \lambda_{r}\right) \in E^{r} \mid 1 \leq \lambda_{1}<\lambda_{2}<\right.$ $\left.\cdots<\lambda_{r} \leq n\right\} \rightarrow\{-1,0,+1\}$ such that $\chi\left(i_{1}, i_{2}, \ldots, i_{r}\right)=\operatorname{sign} \operatorname{det}\left(v_{i_{1}}, v_{i_{2}}, \ldots, v_{i_{r}}\right)$ the (affine) chirotope of $\mathcal{P}$. Let $\left[i_{1} i_{2} \cdots i_{r}\right]=\chi\left(i_{1}, i_{2}, \ldots, i_{r}\right)$. Clearly, for example, the set $\left\{p_{i_{1}}<p_{i_{2}}<\cdots<p_{i_{r}}\right\}$ is in general position if and only if $\left[i_{1} i_{2} \cdots i_{r}\right] \neq 0$. In this paper this will always be the case: we say the chirotope is uniform. Also, given a set $W$ of $r+1$ vectors in $\mathbb{R}^{r}, w_{1}, w_{2}, \ldots, w_{r+1}$, then $\operatorname{det}\left(\hat{w}_{1}, w_{2}, \ldots, w_{r+1}\right) w_{1}-$ $\operatorname{det}\left(w_{1}, \hat{w}_{2}, \ldots, w_{r+1}\right) w_{2}+\cdots+(-1)^{r} \operatorname{det}\left(w_{1}, w_{2}, \ldots, \hat{w_{r+1}}\right) w_{r+1}$ has for the $j$ th coordinate $0=\operatorname{det}\left(\left(w_{1}^{j}, w_{1}\right),\left(w_{2}^{j}, w_{2}\right), \ldots,\left(w_{r+1}^{j}, w_{r+1}\right)\right)$. Let, for such a subset $\left\{p_{i_{1}}, p_{i_{2}}\right.$, $\left.\ldots, p_{i_{r+1}}\right\}$ of $\mathcal{P}, \underline{C}$ be the set of indices of its elements. $\underline{C}=C^{+} \cup C^{-}$, where $C^{ \pm}=\{j \in$ $\left.C \mid(-1)^{j-1}\left[i_{1} i_{2} \cdots \hat{i_{j}} \cdots i_{r+1}\right]= \pm 1\right\}$; this is the signature of the circuit $C=\left(C^{+}, C^{-}\right)$ determined by the chirotope. We also consider as a signed circuit $-C=\left(C^{-}, C^{+}\right)$. Since the first coordinates of the vectors $v_{i}$ are always one, there will be no positive circuits, that is, circuits with $C^{-}=\emptyset$. The oriented matroid is then said to be acyclic. We proceed now with the general definitions.

As usual, we view $\Lambda(n, r)=\left\{\left[\lambda_{1} \lambda_{2} \cdots \lambda_{r}\right] \mid 1 \leq \lambda_{1}<\lambda_{2}<\cdots<\lambda_{r} \leq n\right\}$ as a (free) set of variables, and adopt the following notation for the ideal $\mathbb{R}[\Lambda(n, r)]$ : $\left[\lambda_{\pi(1)} \lambda_{\pi(2)} \cdots \lambda_{\pi(r)}\right]=\operatorname{sign}(\pi) \cdot\left[\lambda_{1} \lambda_{2} \cdots \lambda_{r}\right]$ if $\pi:\{1,2, \ldots, r\} \rightarrow\{1,2, \ldots, r\}$ is a permutation (of $\operatorname{sign}(\pi)$ ) and zero otherwise. The three-term Graßmann-Plücker polynomials are the polynomials of form

$$
\begin{aligned}
\left\{\alpha_{1} \alpha_{2} \cdots \alpha_{r-2} \mid \beta_{1} \beta_{2} \beta_{3} \beta_{4}\right\}:= & {\left[\alpha_{1} \alpha_{2} \cdots \alpha_{r-2} \beta_{1} \beta_{2}\right] \cdot\left[\alpha_{1} \alpha_{2} \cdots \alpha_{r-2} \beta_{3} \beta_{4}\right] } \\
& -\left[\alpha_{1} \alpha_{2} \cdots \alpha_{r-2} \beta_{1} \beta_{3}\right] \cdot\left[\alpha_{1} \alpha_{2} \cdots \alpha_{r-2} \beta_{2} \beta_{4}\right] \\
& +\left[\alpha_{1} \alpha_{2} \cdots \alpha_{r-2} \beta_{1} \beta_{4}\right] \cdot\left[\alpha_{1} \alpha_{2} \cdots \alpha_{r-2} \beta_{2} \beta_{3}\right],
\end{aligned}
$$

for $\left(\alpha_{1}, \ldots, \alpha_{r-2}\right) \in E^{r-2}$ and $\left(\beta_{1}, \ldots, \beta_{4}\right) \in E^{4}$.

Definition 2.1. A uniform, rank $r$ chirotope is an assignment $\chi: \Lambda(n, r) \rightarrow\{-1,+1\}$ such that, for every three-term Graßmann-Plücker polynomial, not all of the three corresponding terms are equal (i.e. the set of three terms equals $\{-1,+1\}$ ); furthermore, it is acyclic if for every subset $\left\{i_{1}, \ldots, i_{r+1}\right\}$ of $E$, the $r+1$ signs in $\left\{(-1)^{j-1}\left[i_{1} i_{2} \cdots \hat{i_{j}} \ldots\right.\right.$ 
$\left.\left.i_{r+1}\right] \mid j=1,2, \ldots, r+1\right\}$ are not the same, or, again, this set equals $\{-1,+1\}$. $E=\{1,2, \ldots, n\}$ is the ground set of $\chi$.

We note that consideration of only three-term Graßmann-Plücker polynomials in the previous definition is sufficient because the chirotope is uniform [14], [6], [9]. An equivalent definition of a chirotope, of a more algebraic nature, was given in [9] and [6] in roughly the following terms:

Definition 2.2. Consider a polynomial of monomials $M_{0}, M_{1}, \ldots, M_{p}, P=M_{0}+$ $M_{1}+\cdots+M_{p} \in \mathbb{K}[X]$. We define:

$$
\begin{aligned}
\operatorname{odd}(P) & =2^{-p} \sum_{\substack{0 \leq i_{1}<i_{2}<\cdots<i_{k} \leq p \\
k \text { odd }}} M_{i_{0}} M_{i_{1}} \cdots M_{i_{k}}=2^{-p-1}\left(\prod_{i=0}^{p}\left(1+M_{i}\right)-\prod_{i=0}^{p}\left(1-M_{i}\right)\right), \\
\operatorname{even}(P) & =2^{-p} \sum_{\substack{0 \leq i_{1} i_{i}<\cdots<i_{k} \leq p \\
k \text { even }}} M_{i_{0}} M_{i_{1}} \cdots M_{i_{k}}=2^{-p-1}\left(\prod_{i=0}^{p}\left(1+M_{i}\right)+\prod_{i=0}^{p}\left(1-M_{i}\right)\right) .
\end{aligned}
$$

Note that if $M_{i}(x)= \pm 1$ for all $i=0,1, \ldots, p$, then $\operatorname{odd}(P)(x)$ is either 0 or \pm 1 (and even $(P)(x)$ is either 0 or +1$)$. The first case occurs exactly when $\{-1,+1\}=$ $\left\{M_{0}(x), M_{1}(x), \ldots, M_{p}(x)\right\}$. Clearly, then a uniform chirotope of rank $r$ can be defined as a function $\chi: \Lambda(n, r) \rightarrow\{-1,+1\}$ such that, for every $\left(\alpha_{1}, \ldots, \alpha_{r-2}\right) \in E^{r-2}$ and $\left(\beta_{1}, \ldots, \beta_{4}\right) \in E^{4}$,

$$
\operatorname{odd}\left(\left\{\alpha_{1} \alpha_{2} \cdots \alpha_{r-2} \mid \beta_{1} \beta_{2} \beta_{3} \beta_{4}\right\}\right)(\chi)=0
$$

or, equivalently, even $\left(\left\{\alpha_{1} \alpha_{2} \cdots \alpha_{r-2} \mid \beta_{1} \beta_{2} \beta_{3} \beta_{4}\right\}\right)(\chi)=0$. Similarly, $\chi$ is acyclic provided

$$
\operatorname{odd}\left(\sum_{j=1}^{r+1}(-1)^{j-1}\left[i_{1} i_{2} \cdots \hat{i_{j}} \cdots i_{r+1}\right]\right)(\chi)=0 .
$$

From this point onward, all oriented matroids considered are assumed uniform and acyclic.

As usual, a (abstract, finite) simplicial complex is a pair $\mathcal{K}=(X, \Phi)$ where $X$ is an ordered finite set $(X=E=\{1,2, \ldots, n\}$ in the case of an oriented matroid over $E$ ) and $\Phi$ is a collection of non-empty subsets of $X$ such that:

(i) for all $x \in X,\{x\} \in \Phi$;

(ii) if $\sigma \in \Phi$ and $\sigma^{\prime} \subset \sigma$, then $\sigma^{\prime} \in \Phi . \sigma^{\prime}$ is a face of $\sigma$.

An (ordered) element $\sigma$ of $\Phi$ is a $p$-simplex or a simplex of dimension $p$ if $p=|\sigma|-1$; we distinguish the simplex $\sigma=\left(x_{0} x_{1} \cdots x_{p}\right)$ from its support, $\|\sigma\|=\left\{x_{0}, x_{1}, \ldots, x_{p}\right\}$. The dimension of a complex is the maximum dimension of its simplices and $\mathcal{K}^{p}$ is the set of $p$-simplices of $\mathcal{K}$. In the case of a complex in an oriented matroid of rank $r$, 
we consider that $r>\operatorname{dim} \mathcal{K}$ so that, in a uniform oriented matroid, for example, the simplices are always independent.

Now, we define:

Definition 2.3. Let $\sigma=\left(x_{0} x_{1} \cdots x_{p}\right)$ and $\tau=\left(y_{0} y_{1} \cdots y_{q}\right)$ be two support-disjoint simplices (i.e. $\|\sigma\| \cap\|\tau\|=\emptyset$ ) in an oriented matroid $\mathcal{O}$ of rank $r$ with $p+q=r-1$; the intersection number of $\tau$ relatively to $\sigma$ is

$$
\begin{aligned}
\mathcal{I}(\sigma, \tau)= & (-1)^{p} \text { odd }\left(\sum_{i=0}^{p}(-1)^{i}\left[x_{0} \cdots \hat{x}_{i} \cdots x_{p} y_{0} \cdots y_{q}\right]\right) \\
& \cdot \operatorname{even}\left(\sum_{j=0}^{q}(-1)^{j+p+1}\left[x_{0} \cdots x_{p} y_{0} \cdots \hat{y}_{j} \cdots y_{q}\right]\right) .
\end{aligned}
$$

We say $\mathcal{O}$ is admissible with respect to a complex $\mathcal{K}=(X, \Phi)$ if, for all support-disjoint simplices $\sigma, \tau \in \Phi$ with the proper dimensions, $\mathcal{I}(\sigma, \tau)=0$.

A p-chain in an oriented matroid $\mathcal{O}$ is a function $c: \mathcal{K}^{p} \rightarrow \mathbb{Z}$, written as usual as the (formal) sum $c=\sum_{\sigma \in \mathcal{K}^{p}} c(\sigma) \sigma$, such that any two faces of two different elements of $c^{-1}(\mathbb{Z} \backslash\{0\})$ (with the proper dimensions) do not intersect. The support of $c$ is $\|c\|=$ $\bigcup_{c(\sigma) \neq 0}\|\sigma\|$. We may define boundary, cycle and homology groups as usual: for example, $\partial\left(x_{0} \cdots x_{p}\right)=\partial_{p}\left(x_{0} \cdots x_{p}\right)=\sum_{i=0}^{p}(-1)^{i}\left(x_{0} \cdots \hat{x}_{i} \cdots x_{p}\right)$, and so $\partial \circ \partial=\partial_{p} \circ \partial_{p-1}=0$, etc. Of course the boundary of a chain in an oriented matroid $\mathcal{O}$ is again in $\mathcal{O}$, and is a cycle.

Since $\mathcal{O}$ is acyclic and uniform, $\mathcal{I}(\sigma, \tau) \neq 0$ if and only if there is a circuit $C$ with $C^{+}=\sigma$ and $C^{-}=\tau$. This explains the second part of Definition 2.3 (see [7]). We may extend this number to chains in a natural way:

Definition 2.4. Let $x=\sum_{\sigma \in S} c(\sigma) \sigma$ and $y=\sum_{\tau \in T} c(\tau) \tau$ be a $p$-chain and a $q$ chain, respectively, in an oriented matroid of rank $r$, with $r=p+q+1$. We define

$$
\mathcal{I}(x, y)=\sum_{\substack{\sigma \in S \\ \tau \in T}} c(\sigma) c(\tau) \mathcal{I}(\sigma, \tau)
$$

Definition 2.5. Let $z_{1}$ and $z_{2}$ be two support-disjoint cycles in $\mathcal{O}$ of dimensions $p$ and $q$, respectively, such that $p+q=r-2$ and $z_{1}=\partial x_{1}$ and $z_{2}=\partial x_{2}$ for a $(p+1)$-chain $x_{1}$ and a $(q+1)$-chain $x_{2}$ in $\mathcal{O}$. We define the linking number of $z_{1}$ and $z_{2}$, denoted by $\left[\left[z_{1}, z_{2}\right]\right]$, to be the integer number $\mathcal{I}\left(x_{1}, z_{2}\right)$ (it will be shown in Theorem 3.2 that this number does not depend on the choice of $\left.x_{1}\right)$.

In the next section we prove that some of the essential properties [18] of the intersection number in Euclidean spaces are still valid in this setting. We use the following results, which are proved in a different section: 
Lemma 2.6. For every integer $p>0$ and every $y_{0}, y_{1}, \ldots, y_{p+1} \in\{-1,+1\}$,

$$
\begin{aligned}
\operatorname{odd}\left(\sum_{i=0}^{p+1} y_{i}\right) & =2^{-1}\left(\operatorname{odd}\left(\sum_{i=0}^{p} y_{i}\right)+y_{p+1} \cdot \operatorname{even}\left(\sum_{i=0}^{p} y_{i}\right)\right) \\
& =y_{k} \cdot \operatorname{even}\left(\sum_{i=0}^{p+1} y_{i}\right) \quad \text { for all } k=0,1, \ldots, p+1 \\
\operatorname{even}\left(\sum_{i=0}^{p+1} y_{i}\right) & =2^{-1}\left(\operatorname{even}\left(\sum_{i=0}^{p} y_{i}\right)+y_{p+1} \cdot \operatorname{odd}\left(\sum_{i=0}^{p} y_{i}\right)\right) \\
& =y_{k} \cdot \operatorname{odd}\left(\sum_{i=0}^{p+1} y_{i}\right) \quad \text { for all } \quad k=0,1, \ldots, p+1 .
\end{aligned}
$$

Lemma 2.7. Let $p$ be an integer greater than 1 , and suppose that $a_{i}= \pm 1, x_{i j}= \pm 1$ and $x_{j i}=x_{i j}$ for every $0 \leq i<j \leq p$, and that, for all $\alpha=\left(i_{0}, i_{1}, i_{2}\right)$ with $0 \leq i_{0}<$ $i_{1}<i_{2} \leq p$,

$$
a_{i_{0}} x_{i_{0} i_{1}} x_{i_{0} i_{2}}-a_{i_{1}} x_{i_{0} i_{1}} x_{i_{1} i_{2}}+a_{i_{2}} x_{i_{0} i_{2}} x_{i_{1} i_{2}}=a_{i_{0}} a_{i_{1}} a_{i_{2}} .
$$

Then, for every $j=0,1, \ldots, p$ :

(i) If $p$ is odd,

$$
\sum_{i=0}^{j-1}(-1)^{i} a_{i} \prod_{\substack{k=0 \\ k \neq i, j}}^{p} x_{i k}+\sum_{i=j+1}^{p}(-1)^{i-1} a_{i} \prod_{\substack{k=0 \\ k \neq i, j}}^{p} x_{i k}=\prod_{\substack{k=0 \\ k \neq j}}^{p} a_{k}
$$

(ii) If $p$ is odd,

$$
\sum_{i=0}^{j-1}(-1)^{i} \prod_{\substack{k=0 \\ k \neq i}}^{p} x_{i k}+\sum_{i=j+1}^{p}(-1)^{i-1} \prod_{\substack{k=0 \\ k \neq i}}^{p} x_{i k}=\prod_{\substack{k=0 \\ k \neq j}}^{p} x_{j k}
$$

(iii)

$$
\operatorname{odd}\left(\sum_{i=0}^{p}(-1)^{i} a_{i}\right)=\sum_{i=0}^{p}(-1)^{i} a_{i} \operatorname{even}\left(\sum_{j=0}^{i-1}(-1)^{j} x_{i j}+\sum_{j=i+1}^{p}(-1)^{j-1} x_{i j}\right) .
$$

Remark 2.8. In the beginning of the proof of part (ii) of Theorem 3.1 we shall see that (1) - the Graßmann-Plücker relations_ can be written for uniform oriented matroids in the form:

$$
a_{i_{0}} x_{i_{0} i_{1}} x_{i_{0} i_{2}}-a_{i_{1}} x_{i_{0} i_{1}} x_{i_{1} i_{2}}+a_{i_{2}} x_{i_{0} i_{2}} x_{i_{1} i_{2}}=a_{i_{0}} a_{i_{1}} a_{i_{2}} .
$$




\section{Properties}

We state and prove in this setting some of the properties valid in Euclidean space [18].

Theorem 3.1. Let $\mathcal{O}$ be a (uniform, acyclic) oriented matroid of rank $r$ and let $x$ and $y$ be a p-chain and a q-chain, respectively, in $\mathcal{O}$. Then:

(i) $\mathcal{I}(x, y)=(-1)^{p q} \mathcal{I}(y, x)$ if $p+q=r-1$;

(ii) $\mathcal{I}(\partial x, y)=(-1)^{p} \mathcal{I}(x, \partial y)$ if $p+q=r$.

Proof. By linearity, it is clearly sufficient to prove both properties in case the chains are simplices.

(i) Let $\sigma=(0 \cdots p)$ and $\tau=(p+1 \cdots r)(r=p+q+1)$. If $\mathcal{I}(\sigma, \tau)=0$, then $\mathcal{I}(\tau, \sigma)=0$ also, by Lemma 2.6, and if $\mathcal{I}(\sigma, \tau)= \pm 1$, then points 0 and $r$ will have opposite signs in the circuit $\underline{\mathrm{C}}=\{0,1, \ldots, r\}$, and hence

$$
[12 \cdots r](-1)^{r}[01 \cdots r-1]=-1 .
$$

By the parity of the functions odd and even and by Lemma 2.6, now,

$$
\begin{aligned}
\mathcal{I}(\sigma, \tau)= & (-1)^{p} \operatorname{even}\left(\sum_{j=p+1}^{r}(-1)^{j}[0 \cdots p p+1 \cdots \hat{\jmath} \cdots r]\right) \\
& \cdot \operatorname{odd}\left(\sum_{i=0}^{p}(-1)^{i}[0 \cdots \hat{\imath} \cdots p p+1 \cdots r]\right) \\
= & (-1)^{p+1}(-1)^{q(p+1)} \operatorname{odd}\left(\sum_{j=p+1}^{r}(-1)^{j}[p+1 \cdots \hat{\jmath} \cdots r 0 \cdots p]\right) \\
& \cdot \operatorname{even}\left(\sum_{i=0}^{p}(-1)^{i}[p+1 \cdots r 0 \cdots \hat{\imath} \cdots p]\right) \quad \text { by (3)) } \\
= & (-1)^{p q+q} \text { odd }\left(\sum_{j=p+1}^{r}(-1)^{j-p-1}[p+1 \cdots \hat{\jmath} \cdots r 0 \cdots p]\right) \\
& \cdot \operatorname{even}\left(\sum_{i=0}^{p}(-1)^{i+q+1}[p+1 \cdots r 0 \cdots \hat{\imath} \cdots p]\right) \\
= & (-1)^{p q} \mathcal{I}(\tau, \sigma) .
\end{aligned}
$$

(ii) Let $\sigma=(0 \cdots p)$ and $\tau=(p+1 \cdots r+1)(r=p+q)$ and set $b_{i j}:=$ $[01 \cdots \hat{\imath} \cdots \hat{\jmath} \cdots r+1]$ for $0 \leq i<j \leq r+1$. For $p+1 \leq j_{0}<j_{1}<j_{2} \leq r+1$, let $p+1 \leq k_{1}<k_{2}<\cdots<k_{q-2} \leq r+1$ be such that $\left\{j_{0}, j_{1}, j_{2}\right\} \cup\left\{k_{1}, k_{2}, \ldots, k_{q-2}\right\}=$ $\{p+1,1, \ldots, r+1\}$. With this notation, by (1), we have

$\operatorname{odd}\left(\left\{01 \cdots \hat{\imath} \cdots p k_{1} k_{2} \cdots k_{q-2} \mid j_{0} j_{1} j_{2} i\right\}\right)=\operatorname{odd}\left(b_{i j_{2}} b_{j_{0} j_{1}}-b_{i j_{1}} b_{j_{0} j_{2}}+b_{i j_{0}} b_{j_{1} j_{2}}\right)=0$ 
or, equivalently (by multiplying all terms by $b_{j_{0} j_{1}} b_{j_{0} j_{2}} b_{j_{1} j_{2}}$ and cancelling squares),

$$
b_{i j_{0}} b_{i j_{1}} b_{i j_{2}}=b_{i j_{2}} b_{j_{0} j_{2}} b_{j_{1} j_{2}}-b_{i j_{1}} b_{j_{0} j_{1}} b_{j_{1} j_{2}}+b_{i j_{0}} b_{j_{0} j_{1}} b_{j_{0} j_{2}} .
$$

Hence, we may apply Lemma 2.7(iii) as done below:

$$
\begin{aligned}
\mathcal{I}(\partial \sigma, \tau)= & \sum_{m=0}^{p}(-1)^{m} \mathcal{I}((0 \cdots \hat{m} \cdots p),(p+1 \cdots r+1)) \\
= & \sum_{m=0}^{p}(-1)^{m+p-1} \text { odd }\left(\sum_{i=0}^{m-1}(-1)^{i} b_{i m}+\sum_{i=m+1}^{p}(-1)^{i-1} b_{m i}\right) \\
& \cdot \operatorname{even}\left(\sum_{j=p+1}^{r+1}(-1)^{j-1} b_{m j}\right) \\
= & (-1)^{p-1} \sum_{m=0}^{p}(-1)^{m}(-1)^{r} b_{m r+1} \text { odd }\left(\sum_{i=0}^{m-1}(-1)^{i} b_{i m}+\sum_{i=m+1}^{p}(-1)^{i-1} b_{m i}\right) \\
& \cdot \text { odd }\left(\sum_{j=p+1}^{r+1}(-1)^{j-1} b_{m j}\right) \quad \text { (by Lemma 2.6). }
\end{aligned}
$$

Applying now Lemma 2.7(iii),

$$
\begin{aligned}
\mathcal{I}(\partial \sigma, \tau)= & (-1)^{p-1}\left[\sum_{m=0}^{p}(-1)^{m}(-1)^{r} b_{m r+1}\right. \\
\left.\cdot \text { odd }\left(\sum_{i=0}^{m-1}(-1)^{i} b_{i m}+\sum_{i=m+1}^{p}(-1)^{i-1} b_{m i}\right)\right] & \cdot\left[\sum_{j=p+1}^{r+1}(-1)^{j-1} b_{m j} \operatorname{even}\left(\sum_{k=p+1}^{j-1}(-1)^{k} b_{k j}+\sum_{k=j+1}^{r+1}(-1)^{k-1} b_{j k}\right)\right] \\
= & (-1)^{p} \sum_{j=p+1}^{r+1}(-1)^{j-1} \\
& \cdot\left[\sum_{m=0}^{p}(-1)^{m} b_{m j} \text { even }\left(\sum_{i=0}^{m-1}(-1)^{i} b_{i m}+\sum_{i=m+1}^{p}(-1)^{i-1} b_{m i}\right)\right] \\
& \cdot \operatorname{even}\left(\sum_{k=p+1}^{j-1}(-1)^{k} b_{k j}+\sum_{k=j+1}^{r+1}(-1)^{k-1} b_{j k}\right) \quad \text { (by (3)) } \\
= & (-1)^{p}(-1)^{p} \sum_{j=p+1}^{r+1}(-1)^{j-p-1} \text { odd }\left(\sum_{m=0}^{p}(-1)^{m} b_{m j}\right) \\
& \cdot \operatorname{even}\left(\sum_{k=p+1}^{j-1}(-1)^{k} b_{k j}+\sum_{k=j+1}^{r+1}(-1)^{k-1} b_{j k}\right) \quad \text { (by Lemma 2.7(iii)) } \\
= & (-1)^{p} \mathcal{I}(\sigma, \partial \tau) .
\end{aligned}
$$


Theorem 3.2. Let $\mathcal{O}$ be a (uniform, acyclic) oriented matroid of rank $r$. Let $z_{1}=\partial x_{1}$ and $z_{2}=\partial x_{2}$ be two support-disjoint cycles in $\mathcal{O}$ with $\operatorname{dim} z_{1}=p, \operatorname{dim} z_{2}=q$ and $p+q=r-2$. Then:

(i) If also $z_{1}=\partial y_{1}$ for $a(p+1)$-chain $y_{1}$, then $\mathcal{I}\left(x_{1}, z_{2}\right)=\mathcal{I}\left(y_{1}, z_{2}\right)$ (see Definition 2.5);

(ii) $\left[\left[z_{1}, z_{2}\right]\right]=(-1)^{p q+1}\left[\left[z_{2}, z_{1}\right]\right]$.

Proof. By Theorem 3.1, we have:

(i) $\mathcal{I}\left(x_{1}, \partial x_{2}\right)-\mathcal{I}\left(y_{1}, \partial x_{2}\right)=\mathcal{I}\left(x_{1}-y_{1}, \partial x_{2}\right)=(-1)^{p+1} \mathcal{I}\left(\partial\left(x_{1}-y_{1}\right), x_{2}\right)=0 ;$

$$
\begin{aligned}
{\left[\left[z_{1}, z_{2}\right]\right] } & =\mathcal{I}\left(x_{1}, \partial x_{2}\right)=(-1)^{q(p+1)} \mathcal{I}\left(\partial x_{2}, x_{1}\right) \\
& =(-1)^{q(p+1)}(-1)^{q+1} \mathcal{I}\left(x_{2}, \partial x_{1}\right)=(-1)^{p q+1}\left[\left[z_{2}, z_{1}\right]\right] .
\end{aligned}
$$

\section{On Brehm's Triangulation of the Möbius Strip}

For this section, we again need a technical result that we prove in the next section:

Lemma 4.1. For every pair $(\sigma, \tau)$ of disjoint simplices in an oriented matroid $\mathcal{O}$ of rank $r$ such that $r=\operatorname{dim} \sigma+\operatorname{dim} \tau, \mathcal{I}(\sigma, \partial \tau)=[[\partial \sigma, \partial \tau]]$ is either 0 or \pm 1. If $r>3$ is even and $A$ is a subset of $E$ with $n=r+2$ elements, then there are two simplices $\sigma$ and $\tau$ of dimensions greater than 1 such that $A=\|\sigma\| \dot{\cup}\|\tau\|$ and $[[\partial \sigma, \partial \tau]] \neq 0$.

Remark 4.2. For any given rank, the restriction of $\mathcal{O}$ to the set of vertices involved is realizable - as the dual of a (clearly realizable) rank 2 oriented matroid. This is used implicitly in our proof.

One advantage of our (algebraic) definition is the possibility of handling "automatic proofs". Similar to [4], for example, the proof of the previous lemma in rank 4 can be done by showing (with a computer) that a given polynomial $p$ belongs to the ideal generated in $\mathbb{K}[\Lambda(6,4)]$ (where $\mathbb{K}=\mathbb{R}$ or $\mathbb{K}=\mathbb{Z} / 3 \mathbb{Z}$ ) by the polynomials that appear in (1) and (2) and by the polynomials of form $\left[\lambda_{1} \lambda_{2} \cdots \lambda_{r}\right]^{2}-1$, since all considered chirotopes are zeros of all of them, and hence of $p$. We did this using Mathematica, showing:

(i) At least one of the edges in $\partial \tau$ does not intersect $\sigma$; in this case, the polynomial shown to be in the ideal is, in the notation above, $p=\prod_{i=1}^{3} \mathcal{I}\left(\sigma, \tau^{i}\right)$. This is a clear consequence of the proof of the first part of the previous lemma.

(ii) If two of these edges intersect $\sigma$, the intersection numbers are opposite; in this case, $p=\left(\mathcal{I}\left(\sigma, \tau^{1}\right)+\mathcal{I}\left(\sigma, \tau^{3}\right)\right)\left(\mathcal{I}\left(\sigma, \tau^{1}\right)-\mathcal{I}\left(\sigma, \tau^{2}\right)\right)\left(-\mathcal{I}\left(\sigma, \tau^{2}\right)+\mathcal{I}\left(\sigma, \tau^{3}\right)\right)$.

(iii) From the ten different ways of splitting $E=\{1,2, \ldots, 6\}$ into two sets of cardinal 3 (two complementary triangles), in at least one of them the triangles do link. Now,

$$
p=\prod_{1<i<j \leq 6}\left(1-[[(1 i j), \overline{(1 i j)}]]^{2}\right), \quad \text { where }\|\overline{(1 i j)}\|=E \backslash\{1, i, j\} .
$$




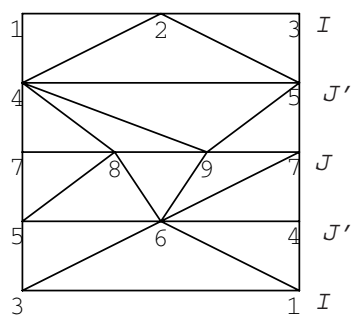

Fig. 1

Let us consider Brehm's triangulation [8] of the Möbius strip, $\mathcal{K}=([1,9], \Phi)$ represented in Fig. 1; we denote by $I$ the closed curve $\left(\begin{array}{ll}1 & 2\end{array}\right)-\left(\begin{array}{ll}1 & 3\end{array}\right)+\left(\begin{array}{ll}2 & 3\end{array}\right)$ (the border of a simplex not in $\mathcal{K}$, (123)), and by $J$ and $J^{\prime}$ the curves $\partial(789)$ and $\partial(456)$. We prove the following:

Theorem 4.3. There is no acyclic, uniform, rank 4 oriented matroid admissible with respect to $\mathcal{K}$, and hence $[8] \mathcal{K}$ is not geometrically embeddable.

Proof. Contrary to our claim, we assume there is an oriented matroid $\mathcal{O}$ admissible with $\mathcal{K}$. Then, for every triangle $t$ and for every edge $e$ in $\Phi, \mathcal{I}(t, e)=0$. Our proof is similar to Brehm's proof. See also [17]. We proceed in four steps:

(a) $\left[\left[J, J^{\prime}\right]\right]= \pm 1$. If we exclude from $\Phi$ the triangles containing vertices of label 1 , 2 or 3 , then we are left with the following triangles:

(459), (467), (478), (489) and $\overline{(457)}, \overline{(458)}, \overline{(468)}, \overline{(469)}, \overline{(479)}$,

where by $\bar{A}$ we denote the complement in $\{4,5, \ldots, 9\}$ of $A$. The result follows from Lemma 4.1, which asserts the existence of two complementary triangles with linking number \pm 1 , none of which can be in the list above. They must then be (456) and (789).

(b) $[[J, I]]= \pm 1$. Note that (see Fig. 1) $(12)+(23)$ is homologous to $(14)+(45)-$ (3 5) and $-(13)$ to $-(14)+(35)+(56)-(46)$ through borders of triangles of $\Phi$ that are support-disjoint to $J$. Explicitly,

$$
\begin{aligned}
(12)+(23) & =(14)+(45)-(35)+\partial(124)-\partial(245)+\partial(235), \\
-(13) & =-(14)+(35)+(56)-(46)-\partial(136)+\partial(146)-\partial(356)
\end{aligned}
$$

and hence $J^{\prime}=I+\sum_{i=1}^{6}(-1)^{i} \partial\left(\sigma_{i}\right)$, for given $\sigma_{i} \in \Phi$. Now,

$$
\begin{aligned}
{\left[\left[J, J^{\prime}\right]\right] } & \left.\left.=[[J, I]]+\sum_{i=1}^{6}(-1)^{i}\left[\left[J, \partial\left(\sigma_{i}\right)\right]\right]=[[J, I]]+\sum_{i=1}^{6}(-1)^{i} \mathcal{I}\left(J, \sigma_{i}\right)\right]\right] \\
& =[[J, I]] .
\end{aligned}
$$


(c) $[[I, J]]= \pm 1$. This is Theorem 3.2, where $p=q=1$.

(d) $\left[\left[I, J^{\prime}\right]\right]= \pm 2$. Proceeding as in (b), we note that $J$ is homologous to $-(47)+$ $(45)+(57)$, on the one hand, and to $-(57)+(56)-(46)+(47)$, on the other hand; hence $J^{\prime}$ is homologous to $2 J$ (in $E \backslash\|I\|$ ) and [[I, $\left.\left.J^{\prime}\right]\right]=2[[I, J]]$.

However, (d) contradicts the first part of Lemma 4.1.

\section{Proofs of Technical Results}

Lemma 2.6. For every integer $p>0$ and every $y_{0}, y_{1}, \ldots, y_{p+1} \in\{-1,+1\}$,

$$
\begin{aligned}
\operatorname{odd}\left(\sum_{i=0}^{p+1} y_{i}\right) & =2^{-1}\left(\operatorname{odd}\left(\sum_{i=0}^{p} y_{i}\right)+y_{p+1} \cdot \operatorname{even}\left(\sum_{i=0}^{p} y_{i}\right)\right) \\
& =y_{k} \cdot \operatorname{even}\left(\sum_{i=0}^{p+1} y_{i}\right) \quad \text { for all } k=0,1, \ldots, p+1 \\
\operatorname{even}\left(\sum_{i=0}^{p+1} y_{i}\right) & =2^{-1}\left(\operatorname{even}\left(\sum_{i=0}^{p} y_{i}\right)+y_{p+1} \cdot \operatorname{odd}\left(\sum_{i=0}^{p} y_{i}\right)\right) \\
& =y_{k} \cdot \text { odd }\left(\sum_{i=0}^{p+1} y_{i}\right) \quad \text { for all } k=0,1, \ldots, p+1 .
\end{aligned}
$$

Proof. The first equality follows immediately from Definition 2.2; the second is due to the fact that either all $y_{i}$ are equal (to +1 or to -1$)$, and then odd $\left(\sum_{i=0}^{p+1} y_{i}\right)=y_{0}(=$ $\left.y_{1}=\cdots=y_{p+1}\right)$ and even $\left(\sum_{i=0}^{p+1} y_{i}\right)=1$, or odd $\left(\sum_{i=0}^{p+1} y_{i}\right)=0=\operatorname{even}\left(\sum_{i=0}^{p+1} y_{i}\right)$.

Lemma 2.7. Let $p$ be an integer greater than 1 , and suppose that $a_{i}= \pm 1, x_{i j}= \pm 1$ and $x_{j i}=x_{i j}$ for every $0 \leq i<j \leq p$, and that, for all $\alpha=\left(i_{0}, i_{1}, i_{2}\right)$ with $0 \leq i_{0}<$ $i_{1}<i_{2} \leq p$,

$$
a_{i_{0}} x_{i_{0} i_{1}} x_{i_{0} i_{2}}-a_{i_{1}} x_{i_{0} i_{1}} x_{i_{1} i_{2}}+a_{i_{2}} x_{i_{0} i_{2}} x_{i_{1} i_{2}}=a_{i_{0}} a_{i_{1}} a_{i_{2}} .
$$

Then, for every $j=0,1, \ldots, p$ :

(i) If $p$ is odd,

$$
\sum_{i=0}^{j-1}(-1)^{i} a_{i} \prod_{\substack{k=0 \\ k \neq i, j}}^{p} x_{i k}+\sum_{i=j+1}^{p}(-1)^{i-1} a_{i} \prod_{\substack{k=0 \\ k \neq i, j}}^{p} x_{i k}=\prod_{\substack{k=0 \\ k \neq j}}^{p} a_{k} .
$$

(ii) If $p$ is odd,

$$
\sum_{i=0}^{j-1}(-1)^{i} \prod_{\substack{k=0 \\ k \neq i}}^{p} x_{i k}+\sum_{i=j+1}^{p}(-1)^{i-1} \prod_{\substack{k=0 \\ k \neq i}}^{p} x_{i k}=\prod_{\substack{k=0 \\ k \neq j}}^{p} x_{j k} .
$$


(iii)

$$
\operatorname{odd}\left(\sum_{i=0}^{p}(-1)^{i} a_{i}\right)=\sum_{i=0}^{p}(-1)^{i} a_{i} \text { even }\left(\sum_{j=0}^{i-1}(-1)^{j} x_{i j}+\sum_{j=i+1}^{p}(-1)^{j-1} x_{i j}\right) .
$$

Proof. (i), (ii) For $p=3$ we must have

$$
\begin{aligned}
& a_{0} x_{01} x_{02}-a_{1} x_{01} x_{12}+a_{2} x_{02} x_{12}=a_{0} a_{1} a_{2}, \\
& a_{0} x_{01} x_{03}-a_{1} x_{01} x_{13}+a_{3} x_{03} x_{13}=a_{0} a_{1} a_{3}, \\
& a_{0} x_{02} x_{03}-a_{2} x_{02} x_{23}+a_{3} x_{03} x_{23}=a_{0} a_{2} a_{3}, \\
& a_{1} x_{12} x_{13}-a_{2} x_{12} x_{23}+a_{3} x_{13} x_{23}=a_{1} a_{2} a_{3}
\end{aligned}
$$

and

$$
\begin{aligned}
& x_{01} x_{02} x_{03}-x_{01} x_{12} x_{13}+x_{02} x_{12} x_{23}=x_{03} x_{13} x_{23}, \\
& x_{01} x_{02} x_{03}-x_{01} x_{12} x_{13}+x_{03} x_{13} x_{23}=x_{02} x_{12} x_{23}, \\
& x_{01} x_{02} x_{03}-x_{02} x_{12} x_{23}+x_{03} x_{13} x_{23}=x_{01} x_{12} x_{13}, \\
& x_{01} x_{12} x_{13}-x_{02} x_{12} x_{23}+x_{03} x_{13} x_{23}=x_{01} x_{02} x_{03}
\end{aligned}
$$

Equations (5) are valid by hypothesis and, e.g. the last equation from (6) is obtained by multiplying both members of the first equation of (5) by $a_{0} x_{03}$ (since $a_{0}^{2} x_{03}^{2}=1$ ), both members of the second equation by $a_{1} x_{12}$, of the third by $-a_{2} x_{12}$ and of the fourth by $-a_{3} x_{03}$, and adding them up.

Now, we assume by induction that conditions (i) and (ii) hold for $p$.

We extend both expressions to $p+2$ simultaneously. By multiplying (i) by $a_{p+1} a_{p+2}$, since for $0 \leq i \leq p$,

$$
a_{i} a_{p+1} a_{p+2}=a_{i} x_{i p+1} x_{i p+2}-a_{p+1} x_{i p+1} x_{p+1 p+2}+a_{p+2} x_{i p+2} x_{p+1 p+2},
$$

we have

$$
\begin{aligned}
\prod_{\substack{i=0 \\
i \neq j}}^{p+2} a_{i}= & \sum_{i=0}^{j-1}(-1)^{i} a_{i} a_{p+1} a_{p+2} \prod_{\substack{k=0 \\
k \neq i, j}}^{p} x_{i k}+\sum_{i=j+1}^{p}(-1)^{i-1} a_{i} a_{p+1} a_{p+2} \prod_{\substack{k=0 \\
k \neq i, j}}^{p} x_{i k} \\
= & \sum_{i=0}^{j-1}(-1)^{i} a_{i} x_{i p+1} x_{i p+2} \prod_{\substack{k=0 \\
k \neq i, j}}^{p} x_{i k}+\sum_{i=j+1}^{p}(-1)^{i-1} a_{i} x_{i p+1} x_{i p+2} \prod_{\substack{k=0 \\
k \neq i, j}}^{p} x_{i k} \\
& -a_{p+1} x_{p+1} p\left(\sum_{i=0}^{j-1}(-1)^{i} x_{i p+1} \prod_{\substack{k=0 \\
k \neq i, j}}^{p} x_{i k}+\sum_{i=j+1}^{p}(-1)^{i-1} x_{i p+1} \prod_{\substack{k=0 \\
k \neq i, j}}^{p} x_{i k}\right) \\
& +a_{p+2} x_{p+1}\left(\sum_{i=0}^{j-1}(-1)^{i} x_{i p+2} \prod_{\substack{k=0 \\
k \neq i, j}}^{p} x_{i k}+\sum_{i=j+1}^{p}(-1)^{i-1} x_{i p+2} \prod_{\substack{k=0 \\
k \neq i, j}}^{p} x_{i k}\right)
\end{aligned}
$$


and then

$$
\begin{aligned}
\prod_{\substack{i=0 \\
i \neq j}}^{p+2} a_{i}= & \sum_{i=0}^{j-1}(-1)^{i} a_{i} \prod_{\substack{k=0 \\
k \neq i, j}}^{p+2} x_{i k}+\sum_{i=j+1}^{p}(-1)^{i-1} a_{i} \prod_{\substack{k=0 \\
k \neq i, j}}^{p+2} x_{i k} \\
& -a_{p+1} x_{p+1} p+2(\underbrace{\sum_{i=0}^{j-1}(-1)^{i} \prod_{\substack{k=0 \\
k \neq i, j, p+2}}^{p+2} x_{i k}+\sum_{i=j+1}^{p}(-1)^{i-1} \prod_{\substack{k=0 \\
k \neq i, j, p+2}}^{p+2} x_{i k}}_{(A)}) \\
& +a_{p+2} x_{p+1} p+2 \\
& \sum_{(B)}^{\sum_{i=0}^{j-1}(-1)^{i} \prod_{\substack{k=0 \\
k \neq i, j, p+1}}^{p+2} x_{i k}+\sum_{i=j+1}^{p}(-1)^{i-1} \prod_{\substack{k=0 \\
k \neq i, j, p+1}}^{p+2}} x_{i k}
\end{aligned} .
$$

Now, in $(A)$, we consider the set of indices of $\prod_{k=0, k \neq i, j, p+2}^{p+2} x_{i k}, I=[0, p+$ $2] \backslash\{i, j, p+2\}=:\left\{k_{1}<k_{2}<\cdots<k_{p}\right\}$. If we apply (ii) to $y_{m n}:=x_{k_{m} k_{n}}$ (and to $b_{m}=$ $a_{k_{m}}$ ), taking for $j$ the value $p$, we obtain $\prod_{l=0, l \neq p}^{p} y_{p l}=A=\prod_{k=0, k \neq j, p+1, p+2}^{p+2} x_{p+1 k}$. Similarly, $B=\prod_{k=0, k \neq j, p+1, p+2}^{p+2} x_{p+2 k}$. Hence,

$$
\begin{aligned}
\prod_{\substack{i=0 \\
i \neq j}}^{p+2} a_{i}= & \sum_{i=0}^{j-1}(-1)^{i} a_{i} \prod_{\substack{k=0 \\
k \neq i, j}}^{p+2} x_{i k}+\sum_{i=j+1}^{p}(-1)^{i-1} a_{i} \prod_{\substack{k=0 \\
k \neq i, j}}^{p+2} x_{i k}-a_{p+1} \prod_{\substack{k=0 \\
k \neq j, p+1}}^{p+2} x_{p+1 k} \\
& +a_{p+2} \prod_{\substack{k=0 \\
k \neq j, p+2}}^{p+2} x_{p+2} \sum_{i=0}^{j-1}(-1)^{i} a_{i} \prod_{\substack{k=0 \\
k \neq i, j}}^{p+2} x_{i k}+\sum_{i=j+1}^{p+2}(-1)^{i-1} a_{i} \prod_{\substack{k=0 \\
k \neq i, j}}^{p+2} x_{i k} .
\end{aligned}
$$

By replacing, for $j=0, \ldots, p+2, a_{i}$ by $x_{i j}$ with $i \neq j$, the conditions of the lemma are fulfilled (see (6)) and hence condition (i) holds for $p+2$ and for these values; but this is condition (ii) for $p+2$.

(iii) For $p=2$, the result follows immediately from (4). Now, by induction on $p$, it is sufficient to prove that the new terms appearing in each member, by Lemma 2.6,

$$
(-1)^{p+1} a_{p+1} \text { even }\left(\sum_{i=0}^{p}(-1)^{i} a_{i}\right)
$$

and

$$
\begin{gathered}
(-1)^{p} \sum_{i=0}^{p}(-1)^{i} a_{i} x_{i p+1} \text { odd }\left(\sum_{j=0}^{i-1}(-1)^{j} x_{i j}+\sum_{j=i+1}^{p}(-1)^{j-1} x_{i j}\right) \\
+(-1)^{p+1} a_{p+1} \operatorname{even}\left(\sum_{j=0}^{p}(-1)^{j} x_{j p+1}\right),
\end{gathered}
$$

respectively, are equal. 
We now consider a generic summand of (7) ( $o$ is any odd number not greater than $p+2)$,

$$
(-1)^{p+1} a_{p+1} \prod_{j=1}^{o-1}(-1)^{i_{j}} a_{i_{j}}=(-1)^{i_{1}+i_{2}+\cdots+i_{o-1}+p+1} \prod_{j=1}^{o} b_{j}=s \prod_{j=1}^{o} b_{j},
$$

where $b_{j}=a_{i_{j}}$ for $j=1,2, \ldots, o-1, b_{o}=a_{p+1}$ and $s=(-1)^{i_{1}+i_{2}+\cdots+i_{o-1}+p+1}$, and let $y_{k l}=x_{i_{k} i_{l}}$. By (i) and (ii)

$$
\left.\begin{array}{rl}
s \prod_{j=1}^{o} b_{j}= & s \sum_{j=1}^{o}(-1)^{j-1} b_{j} \prod_{\substack{k=1 \\
k \neq j}}^{o} y_{j k} \\
= & s \sum_{j=1}^{o-1}(-1)^{j-1} b_{j} x_{i_{j} p+1} \prod_{\substack{k=1 \\
k \neq j}}^{o-1} y_{j k}+s a_{p+1} \prod_{k=1}^{o-1} x_{i_{k} p+1} \\
= & (-1)^{p} \sum_{j=1}^{o-1}(-1)^{j}(-1)^{i_{j}} a_{i_{j}} x_{i_{j} p+1} \prod_{\substack{k=1 \\
k \neq j}}^{o-1}(-1)^{i_{k}} x_{i_{j} i_{k}} \\
& +(-1)^{p+1} a_{p+1} \prod_{k=1}^{o-1}(-1)^{i_{k}} x_{i_{k} p+1} \\
= & (-1)^{p} \sum_{j=1}^{o-1}(-1)^{j}(-1)^{i_{j}} a_{i_{j}} x_{i_{j} p+1}\left(\prod_{k=1}^{j-1}(-1)^{i_{k}} x_{i_{j} i_{k}}\right)(-1)^{o-j-1} \\
& \times\left(\prod_{k=j+1}^{o-1}(-1)^{i_{k}-1} x_{i_{j} i_{k}}\right) \\
& +(-1)^{p+1} a_{p+1} \prod_{k=1}^{o-1}(-1)^{i_{k}} x_{i_{k} p+1} .
\end{array}\right\}
$$

Now, the proof ends by noting that (10) is the generic term of (8), and that (11) is the generic term of (9).

Lemma 4.1. For every pair $(\sigma, \tau)$ of disjoint simplices in an oriented matroid $\mathcal{O}$ of rank $r$ such that $r=\operatorname{dim} \sigma+\operatorname{dim} \tau, \mathcal{I}(\sigma, \partial \tau)=[[\partial \sigma, \partial \tau]]$ is either 0 or \pm 1. If $r>3$ is even and $A$ is a subset of $E$ with $n=r+2$ elements, then there are two simplices $\sigma$ and $\tau$ of dimensions greater than 1 such that $A=\|\sigma\| \dot{U}\|\tau\|$ and $[[\partial \sigma, \partial \tau]] \neq 0$.

Proof. (First part) Let $\sigma=\left(\sigma_{0} \cdots \sigma_{p}\right)$ and $\tau=\left(\tau_{0} \cdots \tau_{q}\right)$. By definition, $\mathcal{I}(\sigma, \partial \tau)=$ $\sum_{i=0}^{q}(-1)^{i} \mathcal{I}\left(\sigma, \tau^{i}\right)$, where we denote by $\tau^{i}$ the simplex obtained by deleting the $i$ th vertex in $\tau$. We suppose $\mathcal{I}\left(\sigma, \tau^{i}\right)$ and $\mathcal{I}\left(\sigma, \tau^{j}\right)$ are different from zero for given $0 \leq$ $i<j \leq q$, and consider $\mathcal{I}\left(\tau^{i}, \sigma\right)$, again different from zero by Theorem 3.1. As in the proof of Lemma 2.6, being defined as the product of odd $(P)$ by even $(Q)$ for given 


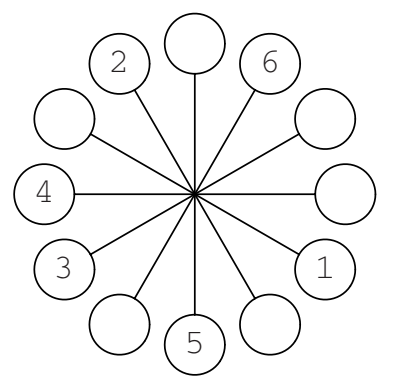

Fig. 2

polynomials $P$ and $Q, \mathcal{I}\left(\tau^{i}, \sigma\right)$ is equal to any of the terms in $P$, which must all be equal. Hence, $\mathcal{I}\left(\tau^{i}, \sigma\right)=(-1)^{q-1}(-1)^{j-1}\left[\tau_{0} \cdots \hat{\tau}_{i} \cdots \hat{\tau}_{j} \cdots \tau_{q} \sigma_{0} \cdots \sigma_{p}\right]$ (one of these terms) and finally

$$
\mathcal{I}\left(\sigma, \tau^{i}\right)=(-1)^{(p+1)(q-1)}(-1)^{j-1}\left[\tau_{0} \cdots \hat{\tau}_{i} \cdots \hat{\tau}_{j} \cdots \tau_{q} \sigma_{0} \cdots \sigma_{p}\right]
$$

In the same way,

$$
\mathcal{I}\left(\sigma, \tau^{j}\right)=(-1)^{(p+1)(q-1)}(-1)^{i}\left[\tau_{0} \cdots \hat{\tau}_{i} \cdots \hat{\tau}_{j} \cdots \tau_{q} \sigma_{0} \cdots \sigma_{p}\right],
$$

and therefore $(-1)^{i} \mathcal{I}\left(\sigma, \tau^{i}\right)+(-1)^{j} \mathcal{I}\left(\sigma, \tau^{j}\right)=0$. This shows the first part of the lemma.

(Second part) Let $\mathcal{O}_{\bar{A}}$ be the restriction of $\mathcal{O}$ to $A=\|\sigma\| \dot{U}\|\tau\|$, and let $\mathcal{O}_{\bar{A}}^{*}$ be the (rank 2) dual.

Since $\mathcal{O}_{\bar{A}}$ is uniform and acyclic, $\mathcal{O}_{\bar{A}}^{*}$ is uniform and totally cyclic [1]. Hence, if we look at $\mathcal{O}_{\bar{A}}^{*}$ as a closed oriented line, like in Fig. 2, where $n=6$, then the six "filled cells" are not consecutive since, for a circuit $C=A \backslash\{x\}$ of $\mathcal{O}_{\bar{A}}, C^{+}$and $C^{-}$are the "two sides" of the hyperplane $\{x\}$ of $\mathcal{O}_{\frac{*}{A}}^{*}$. Since $r$ (and thus $n$ ) is even, they cannot be strictly alternating as well.

Let $\tau^{\prime}$ be a simplex in $\partial \tau$ and suppose $\|\tau\|=\left\|\tau^{\prime}\right\| \cup\{x\}$. Then the fact that $\mathcal{I}\left(\sigma, \tau^{\prime}\right) \neq 0$ means that the hyperplane $\{x\}$ of $\mathcal{O}_{\bar{A}}^{*}$ separates exactly $\|\sigma\|$ of $\left\|\tau^{\prime}\right\|$. For example, in Fig. 2 , $\{2,6\}$ is separated from $\{1,3,5\}$ by $\{4\}$, and from $\{3,4,5\}$ by $\{1\}$.

We prove now that there are always two points in consecutive positions in the oriented line $\mathcal{O}_{\frac{*}{A}}^{*}$, with at least two points in each side of the set formed by all other points.

As we have noted, since $r$ is even, there must be two points, say, $x$ and $y$, occupying consecutive positions. Either $x$ and $y$ already fulfill the previous requirement or all but one point, $z$, lie in the same side of the complement of $\{x, y\}$. We suppose without loss of generality that $z$ stands opposite to $x$ in the cocircuit determined by $\{y\}$ (that is, lays on the opposite half circle). If $z$ is in the last possible position, so that the hyperplanes $\{z\}$ and $\{x\}$ are consecutive, the two points, on the other half circle, closest to $x$ already verify the property, being $x$ and $y$ in one of the sides of the complement and all points but $x, y$ in the other side ( $z$ included); otherwise, there will be a fourth point, $t$, preceding $x$ in the circular ordering, and then $y$ and $z$ are in one side of its complement of $\{x, y\}$ and the remaining points in the other. 


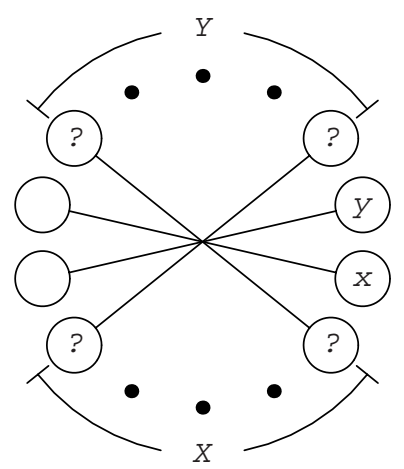

Fig. 3

In Fig. 3, $z$ is in $Y$ and all other points in $X$, and either $z$ occupies the position in $Y$ farthest from $y$ (and $x$ ), or there exists a $t$ in the position in $X$ closest to $x$. Now, we assume that in the oriented line $\mathcal{O}_{\bar{A}}^{*}, x$ and $y$ are in consecutive positions and that in both sides of the complement there are at least two other points. Then let $X \cup Y$ be the complement of $\{x, y\}$ so that the points in circular order in $\mathcal{O}_{\frac{*}{A}}^{*}$ are successively the points of $X, x, y$ and the points of $Y$. Suppose $\|\sigma\|=X \cup\{x\}$ and $\|\tau\|=Y \cup\{y\}$. Then $\mathcal{I}(\sigma, \partial \tau) \neq 0$, since $y$ is the only point $u \in Y \cup\{y\}$ such that $u$ separates $Y \cup\{y\} \backslash\{u\}$ from $X \cup\{x\}$.

\section{References}

1. A. Björner, M. Las Vergnas, B. Sturmfels, N. White and G. Ziegler, Encyclopedia of Mathematics and its Applications 46, Cambridge University Press, Cambridge, 1993.

2. R. Bland and M. Las Vergnas, Orientability of matroids, J. Combin. Theory Ser.B 24(1) (1978), 94-123.

3. J. Bokowski, Private communication, 1999.

4. J. Bokowski and A. Guedes de Oliveira, Invariant theory-like theorems for matroids and oriented matroids, Adv. in Math. 109(1) (1994), 34-44.

5. J. Bokowski and A. Guedes de Oliveira, On the generation of oriented matroids, Discrete Comput. Geom. 24 (2000), 197-208. (Special issue dedicated to Branko Grünbaum), ed. by Gil Kalai and Victor Klee).

6. J. Bokowski, A. Guedes de Oliveira and J. Richter, Algebraic varieties characterizing matroids and oriented matroids, Adv. in Math. 87 (1991), 160-185.

7. J. Bokowski and B. Sturmfels, Computational Synthetic Geometry, Lecture Notes in Mathematics 1355 (1989), Springer-Verlag, Berlin.

8. U. Brehm, A nonpolyhedral triangulated Möbius strip, Proc. Amer. Math. Soc. 89 (1983), 519-522.

9. A. Guedes de Oliveira, Oriented matroids and projective invariant theory, Ph.D. thesis, TH Darmstadt, 1989.

10. B. Grünbaum, Convex Polytopes, Pure and Applied Mathematics 16, Wiley Interscience, New York, 1967.

11. I. Novik, A note on geometric embeddings of simplicial complexes in a Euclidean space, Discrete Comput. Geom. 23(2) (2000), 293-302.

12. L. Gutierrez Novoa, On $n$-ordered sets and order completeness, Pacific J. Math. 15 (1965), 1337-1345.

13. M. Las Vergnas, Bases in oriented matroids, J. Combin. Theory Ser. B 25 (1978), 283-289.

14. M. Las Vergnas, Oriented matroids as signed geometries real in corank 2, in: Finite and Infinite Sets (Proc. 6th Hungarian Combinatorial Conf., Eger, 1981), North-Holland, Amsterdam, 1984, pp. 555-565.

15. J. Lawrence, Oriented matroids and multiply ordered sets, Linear Algebra Appl. 48 (1982), 1-12. 
16. H. Seifert and W. Threlfall, A Textbook of Topology. Seifert, H., Topology of 3-Dimensional Fibered Spaces, (Joan S. Birman and Julian Eisner, eds.). Translated from German. Pure and Applied Mathematics 89, Academic Press, New York, 1980.

17. R. A. Silva, Mergulhos lineares no plano e no espaço, M.Sc. Thesis (2001), University of Porto.

18. Wu Wen-Tsün, A Theory of Imbedding, Immersion and Isotopy of Polytopes in a Euclidean Space, Science Press, Peking, 1965.

Received April 8, 2002, and in revised form March 11, 2003. Online publication December 19, 2003. 